\title{
Reflections on DS/AHP: Lessons to be learnt
}

\author{
Malcolm J. Beynon \\ Cardiff Business School, Cardiff University, \\ Colum Drive, Cardiff, CF10 3EU, Wales, UK \\ BeynonMJecardiff.ac.uk
}

\begin{abstract}
DS/AHP is a technique for multi-criteria decision making (MCDM), based on the Dempster-Shafer Theory of evidence (DST) and the Analytic Hierarchy Process (AHP). Since its introduction it has been developed and applied by a number of authors, as well as form the foundation for other DST related MCDM techniques. This paper reviews the evolution and impact of DS/AHP, culminating in a critical perspective, over relevant criteria, namely i) Ease of understanding, ii) A champion, iii) Software development and iv) Its pertinent development, for its position in the area of MCDM. The critical perspective will include the impacting role DST has had in the evolution of DS/AHP. The lessons learnt, or not learnt, will be of interest to any reader undertaking research with strong influence from DST-based methodologies.
\end{abstract}

Keywords: AHP · Dempster-Shafer Theory · DS/AHP · Reflections

\section{Introduction}

This paper considers the DS/AHP technique for multi-criteria decision making (MCDM) $[1,6]$. The rudiments of DS/AHP are based on the Dempster-Shafer theory (DST) of evidence ([9, 23] - DS part of name) and the Analytic Hierarchy Process ([22] - AHP part of name). The remit of DS/AHP is the preference ranking of decision alternatives (DAs) based over a number of different criteria. From its introduction it has been technically developed $[2,3,4,5,7]$ and applied in real world problems $[17,27,28]$, as well as contributed to the definition of derivative techniques $[10$, $11,13,14]$.

This paper attempts to put into perspective the evolution of the DS/AHP technique, including emphasis on the impact of using DST in its methodology. In the relative short time since its introduction, academic researching/publishing has changed, with other issues beyond just the concomitant publications (associated with the introduction and development of a novel analysis technique) required to be considered. For example, one of these issues is research impact, how the research being undertaken has had impact in a wider context. With this in mind, thoughts on how the evolution of DS/AHP has progressed, from one of its main developers, may resonate similar thoughts with academics using DST in the introduction and development of other novel analysis techniques.

adfa, p. 1, 2011.

(C) Springer-Verlag Berlin Heidelberg 2011 
A critical perspective will develop on the evolution of DS/AHP, rounding on four pertinent criteria to consider, namely, i) Ease of understanding, ii) A champion iii) Software development and iv) Its pertinent development. For the reader, interested in DST, lessons may be learnt from what to keep in perspective when undertaking technique based research with DST.

\section{The DS/AHP}

In this section we briefly describe the DS/AHP technique for MCDM, through an example problem (only basic features of DS/AHP are shown - for a full description see $[1,6]$ and later references). The example concerns the ability of a decision maker (DM) to preference rank eight decision alternatives (DAs), $A, B, C, D, E, F, G$ and $H$ (making up the frame of discernment $\Theta$ ), considered over three criteria, $c_{1}, c_{2}$ and $c_{3}$. The intended goal of the DM is to identify 'Best DA(s)', where DA(s) denotes that more than one DA may be wanted to be identified as best.

With the intention of employing DS/AHP, the DM makes judgements on the preference of identified groups of DAs over the different criteria (preferences relative to $\Theta)$, see Fig. 1.

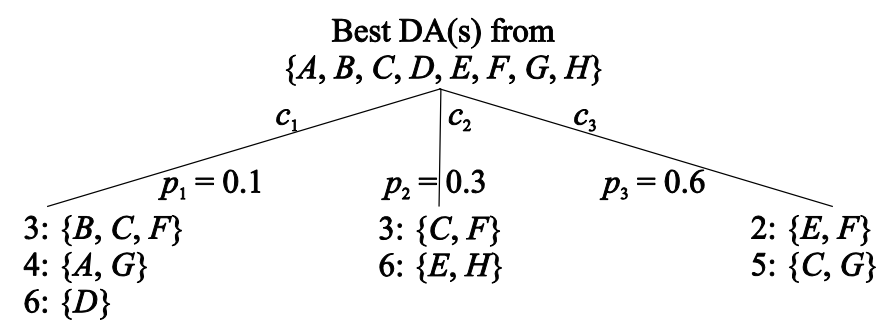

Fig. 1. Preference judgements made on 'Best DAs' MCDM problem

In Fig. 1, three, two and two groups of DAs have been identified for preference on the criteria, $c_{1}, c_{2}$ and $c_{3}$, respectively. Each identified group of DAs have a respective scale value assigned to them, here, a ' 2 to 6 ' scale was adopted, with inference on the identified group of DAs relative to $\Theta$ ranging from moderately preferred (2) to extremely preferred (6), see [2]. Also shown in Fig. 1 are the criterion importance values (CIVs) for the different criteria, $c_{1}: p_{1}=0.1, c_{2}: p_{2}=0.3$ and $c_{3}: p_{3}=0.6$ (found through a weight assignment approach - see [4] for example). From Fig. 1, for the $c_{2}$ criterion, the associated comparison matrix is of the form:

$$
\begin{gathered}
\{C, F\} \\
\{C, F\} \\
\Theta E, H\} \\
\Theta
\end{gathered}\left(\begin{array}{ccc}
1 & 0 & 3 p_{2} \\
0 & 1 & 6 p_{2} \\
1 /\left(3 p_{2}\right) & 1 /\left(6 p_{2}\right) & 1
\end{array}\right),
$$

which gives the respective mass values and focal elements (using general CIV $p_{2}$ ), forming a criterion BOE (body of evidence - made up of the mass values $m_{h}\left(s_{i}\right)=$ 
$a_{i} p /\left(\sum_{j=1}^{d} a_{j} p+\sqrt{d}\right), i=1,2, \ldots d$ and $m_{h}(\Theta)=\sqrt{d} /\left(\sum_{j=1}^{d} a_{j} p+\sqrt{d}\right)$ - see [1]),

defined $m_{2}(\cdot)$, in this case given as:

$$
m_{2}(\{C, F\})=\frac{3 p_{2}}{9 p_{2}+\sqrt{2}}, m_{2}(\{E, H\})=\frac{6 p_{2}}{9 p_{2}+\sqrt{2}} \text { and } m_{2}(\Theta)=\frac{\sqrt{2}}{9 p_{2}+\sqrt{2}} .
$$

These general expressions for the mass values in the criterion BOE $m_{2}(\cdot)$, and the other criterion BOEs $m_{1}(\cdot)$ and $m_{3}(\cdot)$ associated with the $c_{1}$ and $c_{3}$ criteria, are graphically illustrated in Fig. 2.
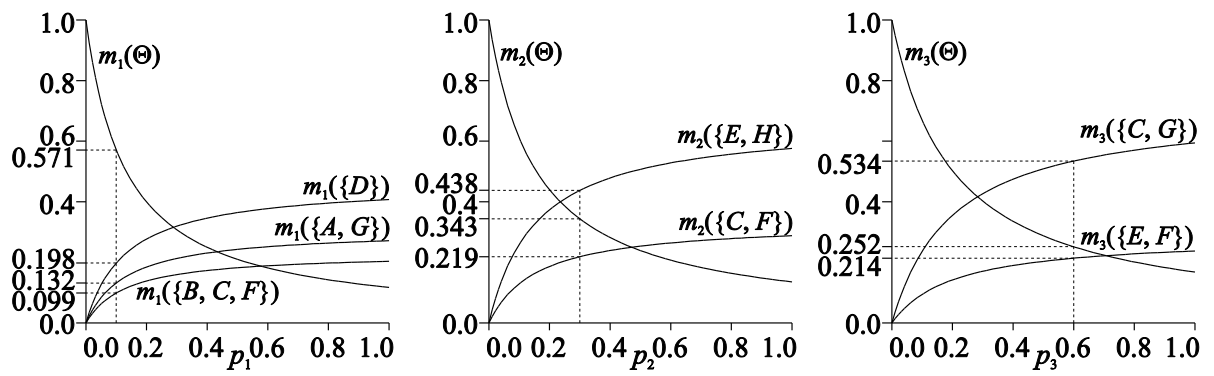

Fig. 2. Mass value graphs of the criterion BOEs, $m_{1}(\cdot), m_{2}(\cdot)$ and $m_{3}(\cdot)$

In Fig. 2, the effect of a CIV ( $p_{i}$ value), on the formulisation of mass values in the criterion BOEs is clearly exposited (as $p_{i} \rightarrow 0$ the majority of the exact belief is assigned to local ignorance $\left(m_{i}(\Theta) \rightarrow 1\right)$ and as $p_{i} \rightarrow 1$ more exact belief is assigned to the identified groups of DAs). Also shown for each criterion are the specific mass values (associated with focal elements) based on their respective CIVs of; $p_{1}=0.1, p_{2}$ $=0.3$ and $p_{3}=0.6$. For the case of the $c_{2}$ criterion, the criterion BOE $m_{2}(\cdot)$ is of the form (with $p_{1}=0.3$ ):

$$
m_{2}(\{C, F\})=0.219, m_{2}(\{E, H\})=0.438 \text { and } m_{2}(\Theta)=0.343 .
$$

Similar results can be found for the other two criteria $\left(m_{1}(\cdot)\right.$ for $c_{1}$ and $m_{3}(\cdot)$ for $\left.c_{3}\right)$. Dempster's combination rule can then be used to combine the evidence from the individual criterion BOEs, resulting in the final BOE, defined $m(\cdot)$, and is of the form:

$$
m(\{C\})=0.177, m(\{D\})=0.031, m(\{E\})=0.098, m(\{F\})=0.071,
$$

$m(\{G\})=0.044, m(\{A, G\})=0.021, m(\{C, F\})=0.068, m(\{C, G\})=0.192$,

$m(\{E, F\})=0.077, m(\{E, H\})=0.115, m(\{B, C, F\})=0.016, m(\Theta)=0.091$.

This final BOE contains all the evidence from the judgements made by a DM over the three criteria. How this evidence is used to identify the most preferred DA or groups of DAs (Best DA(s)) is dependent on how the presence of ignorance is accommodated for. For example, from this final BOE, most preferred groups of DAs can be evaluated, based on the Belief $\left(\operatorname{Bel}\left(s_{i}\right)=\sum_{s_{j} \subseteq s_{i}} m\left(s_{j}\right)\right.$ for all $\left.s_{i} \subseteq \Theta\right)$ and Plausibility $\left(\operatorname{Pls}\left(s_{i}\right)=\sum_{s_{j} \cap s_{i} \neq \varnothing} m\left(s_{j}\right)\right.$ for all $\left.s_{i} \subseteq \Theta\right)$ functions, see Table 1. 
Table 1. Groups of DAs of with largest belief and plausibility values

$\begin{array}{ccc}\text { Size } & \text { Belief - Bel }(\cdot) & \text { Plausibility - Pls }(\cdot) \\ 1 & \{C\}, 0.177 & \{C\}, 0.543 \\ 2 & \{C, G\}, 0.413 & \{C, E\}, 0.833 \\ 3 & \{C, F, G\}, 0.517 & \{C, E, F\}, 0.903 \\ 4 & \{C, E, F, G\}, 0.726 & \{C, E, F, G\}, 0.969\end{array}$

In Table 1, each row identifies the group of DAs, of a certain size, with the largest belief and plausibility values from all other possible groups of DAs of that size. To demonstrate, for a group of DAs of size two, the groups, $\{C, G\}$ and $\{C, E\}$, respectively, were identified as most preferred, based on the belief and plausibility values, respectively.

\section{The development and application of DS/AHP}

Beyond the introductory research on DS/AHP, presented in $[1,6]$, around the year 2000, its early development followed similar developments/issues considered across other MCDM techniques, in particular the AHP, understandably. The impact of the employed preference scale values used and their effect on the results from the employment of DS/AHP was considered in [2], which also looked at possible bounds on the level of ignorance from preference judgements made. There was early effort to present DS/AHP in a software tool, as in [7], which enabled a relatively simple userfriendly utilization of an early version of DS/AHP. [3] incorporated more existing DST formulation, notably involving the non-specificity measure to describe the judgements made and results found using DS/AHP.

Two further developments considered DS/AHP within the group decision making environment, importantly showing the DS/AHP can be used in a multi-level hierarchy in MCDM. [4] considered group decision making, using DS/AHP when there is nonequal importance of the decision makers in the group - using a discount rate formulation developed in DST. [5] considered inter-group alliances and majority rule in group decision making using DS/AHP, using a DST based distance measure to iteratively aggregate the judgements from different members of the decision making group.

Developments away from the original author(s) work on DS/AHP, commenced in 2004 (we believe). [19] developed the DS/AHP approach to handle three types of ambiguous evaluations, termed, missing, interval-valued, and ambiguous lottery evaluations, then applying it to a real estate investment problem. They further suggest future research could conduct psychological experiments to refine and validate models, as well as considering DS/AHP in a fuzzy environment. [20] also developed DS/AHP in creating preference relations of groups of DAs based on their belief intervals, suggesting DS/AHP may not satisfy the transitive property. [21] considered the sensitivity of DS/AHP when using different combination rules (and adding DAs beyond the original DAs included).

[24] furthered the DS/AHP method to collect and aggregate the preferences of multiple DMs, and how this information can then be encoded as weight interval con- 
straints in stochastic multi-criteria acceptability analysis. It should be noted, as suggested in [25], that the main excellent idea underlying the DS/AHP method is in the applying of DST to the AHP, moreover [1,6] proposing to compare groups of DAs by means of their comparisons with the set of all DAs. [29] examined DS/AHP in a majority rule group decision making context (similar investigation to that in [5]). [26] extended DS/AHP, for when there are several hierarchical levels of criteria, they also reduced the computation procedure for the processing and aggregating the incomplete information about criteria and DAs down to solving a finite set of linear programming problems. [15] utilised DS/AHP when comparing results from different combination axioms.

[10] considered DS/AHP in the context of Dezert-Smarandache theory, formulating DSmT/AHP, which included the use of the PCR5 combination rule. In [13], developing DS/AHP, with derivative named technique Belief AHP, took the ability to group DAs together to the criteria level of an MCDM problem, allowing preference judgements to be made also on groups of criteria. Calling it TIN-DS/AHP, [17] developed DS/AHP using the notion of Three-point Interval Number, suggesting it is more inclusive of the complexity of decision problem and fuzziness of human thought.

In terms of applications, [4] applied DS/AHP in a textbook selection problem, using judgements from fellow academics. Incorporating with TOPSIS, [16] applied DS/AHP in deciding on emergency alternatives. The problem of fire extinguishing systems evaluation was considered using DS/AHP in [17]. [27] applied the DS/AHP for decision-making in urban power system planning (they suggest DS/AHP compared with AHP is clear, more tangible, convenient and easy to calculate). [28] applied DS/AHP to enable uncertainty information fusion for flood risk assessment. In a financial context, and part of a bigger research problem, [12] employed DS/AHP to determine the relative importance weights of supply chain performance measures. DS/AHP was applied in [18] to identify attacker type in a game-theoretic approach for preventing threats in intelligent surveillance systems.

It is worthy to note [8] included DS/AHP in their description of the five existing techniques employing evidence theory (DST) in MCDM, suggesting it needed to be tested on large complex problems.

\section{$4 \quad$ DS/AHP Lessons to be learnt?}

This section offers insights into the evolution of DS/AHP. In a general context, it offers evidence of how a technique, heavily based on DST, can find its place amongst other techniques, each potentially offering the same opportunities for analysis, in this case in the area of MCDM. A number of subsections will elaborate on some of the key issues I believe have impacted on its evolution (or not). 


\subsection{Ease of understanding}

The DS/AHP technique, while advocating a clearer or more simplistic approach to MCDM than AHP (as suggested in [20]), or offering a more practical MCDM technique, did have an issue of ease of understanding. During its introduction, while what it could do sounded all very positive, how it actually did it was not as easy to exposit, because of the use of DST possibly, and its own newness.

For the DST literate, I surmise, there was not a problem with understanding the technical rudiments of the technique, whether it made sense in the realm of MCDM was perhaps less assured. However, perhaps where the problem lay was in the MCDM relevant researchers and their ability, or perhaps desire, to work through the various relevant technical issues of DST, including bodies of evidence, mass values and combining evidence etc.

In the early years of its evolution, being an academic in a business school, the early authors' platforms for talking about DS/AHP were mostly through MCDM seminar tracks in Management or Operations Research conferences, where much of the time in seminar presentations was taken up expositing small examples of how DST worked, before then going onto the technique specifically. Clearly, this early exposition meant one cohort of 'potentially interested' researchers got to experience the DS/AHP technique, perhaps a number of them got more benefit from seeing DST in action, possibly for the first time, both initially through small general examples as well as through DS/AHP. However, the DST based academics were never really brought on board in the early years of its introduction, and importantly its development.

This pertinently brings me on to the next issue, namely the championing of the DS/AHP technique.

\subsection{A champion}

From the first introduction of the DS/AHP technique its early evolution was steered by one researcher, a champion so to speak, myself I have to admit, as a person who had helped teach Dempster-Shafer theory in my home university I was keen to use it in some research field. Having an initial 'loose' interest in MCDM I endeavored to see how DST could be employed in some way in MCDM.

Considering AHP from the start, it took a while, but it was possible to construct a development on AHP which was based on DST, and importantly, could bring some advantages over the original AHP (see literature). From the early concomitant research, and spurred on by the success of the first publications $[1,6]$, in regard to DS/AHP I considered its technical development. This followed two directions, firstly, what did AHP have that could be mimicked with DS/AHP, but also what did DST have that could be positively brought into the DS/AHP, bringing further advantages over the original AHP.

While this sounds all very positive, with other commitments and interests the time spent on DS/AHP may not have been enough for its potential to be fully brought out. Further, there were never other full-on champions of the DS/AHP technique, instead the early work on it has been piecemeal by other academics, possibly as part of their 
PhDs etc. Over the years, the notion of a champion has never been full-on, only 'now and again' exposited at conferences. Whether a champion may still materialize we shall wait and see, of a champion of a later derivative of the technique may be what happens.

\subsection{Software development}

One issue relating to the DS/AHP has been the availability of pertinent software that can undertake the relevant MCDM analysis. In [7], some rudimentary software was created, through a summer-employment-scheme of an undergraduate computer science student (one of the co-authors in [7]). The creation of this software was not with the emphasis of getting software out there to be used by other potential users (back then there wasn't the motivation to push this), instead, it was more of an academic exercise in seeing what could be done in terms of how software could bring out the characteristics of DS/AHP, and in-particular the novel features DST brings with it, as well as checking on its usability, if my memory serves me right (in the potential real world).

This I perceive was a missed opportunity in the evolution of DS/AHP, with even rudimentary software 'freely' available to potential users; it would have itself contributed to the championing of the technique. By the nature of the nascence of DS/AHP, it would have been tested in many different applications, with its usability, as well as technical efficiencies/inefficiencies brought to the fore. Moreover, it would have been tested by individuals who may not have had the full working knowledge of the technique (a form of robustness checking). It is a shame that I do not have a running version of this software created early in the evolution of DS/AHP. With other academics working on DS/AHP, I am not aware of freely available DS/AHP software, something that would be of great benefit to its evolution.

Today's increasingly technical world is far different from what was even back in the 2000s, perhaps a smart-phone/ipad app based software approach may be the way forward, something to seriously think about, need to find the time though.

\subsection{Its pertinent development}

Evidenced from section 3, DS/AHP has been developed from its original introduction (in [6]), both from work by the original author of the technique, but also from academics taking independent perspectives on its pertinence/development. It is understandable that any technique, from its initial introduction, will quite possibly be developed in someway, improving its ability to undertake what it is intended for, as well as accessorizing it with the characteristics necessary for other domains it can potentially operate successfully in.

The point here is that how this succession of developments happens is itself a crucial series of moments in the future impact of a technique. That is, especially in the case of academics who work on a technique like DS/AHP, who didn't originally introduce it, they want to put their stamp on it, very often in the form of assigning a derivative name to the resultant development, in this case for example DSmT-AHP 
([23]), Belief AHP ([26, 27]) and TIN-DS/AHP ([24]). There is nothing wrong in doing this, but for the technique in question, or the general methodology underlying the technique, its future impact is fractured into many bits, depending on how many derivative names of derivative versions of the technique have been advocated in the research papers produced.

It may well be that the future impact of DS/AHP will not be ultimately considered/defined under its original name (which only came into being in the second publication on the technique in [1] it has to be said), but under a derivative name, which may have appropriately developed the technique to a successful level of usability, as well as possibly being successful in the other three points outlined in this section of the paper (its championing etc.). Perhaps the accompanying question here is when it is appropriate and advantageous to all concerned to simply add-on developments to a known technique or to attribute a derivative name to the development of the technique. A question that stems from this section, perhaps itself one to consider carefully is, how important, and impacting, is the actual name of a technique to its future impact (survivability) in a problem area.

\section{Conclusions}

This paper has pondered the evolution of the DS/AHP technique for MCDM. Having written the document in the order it is presented, perhaps the first conclusion I have is to not know, or ask the question, on what is success/failure in terms of the evolution/impact of an MCDM based or other technique (based on DST or otherwise). As the early champion of the technique, including being a co-author and single author of the early research publications on DS/AHP, this paper has made me look back and wonder if it has become, or is it where, I hoped it would be. I suppose not, but as mentioned earlier, what is the timeframe to make such judgments in the evolution/impact of a technique.

How to nurture the DS/AHP technique as a viable/popular technique for MCDM is perhaps what we would all like to know. For any technique, especially using DST in line with this conference, are there shackles of tech-ignorance of the DST methodology that shackles any DST-based technique. Further, are the rudiments of DST themselves still evolving, and so there maintains uncertainty in what are the best technical rudiments to employ in DS/AHP?

It may well be that DS/AHP will be known, or its contribution at least, may be that a future technique, with evolved name, will find the optimum position as a popular and versatile technique in the area of MCDM. My final words in this paper are that I may just be missing something on what to do with DS/AHP, I may also be missing the time to put into its championing (or it may not be good enough as a technique for MCDM in its current evolution - who knows). 


\section{References}

1. Beynon, M.: DS/AHP Method: A Mathematical Analysis, including an Understanding of Uncertainty. European Journal of Operational Research. 140(1), 149-165 (2002)

2. Beynon, M.J.: An Investigation of the Role of Scale Values in the DS/AHP Method of Multi-Criteria Decision Making. Journal of Multi-Criteria Decision Analysis. 11(6), 327-343 (2002)

3. Beynon, M.J.: Understanding Local Ignorance and Non-specificity in the DS/AHP Method of Multi-criteria Decision Making. European Journal of Operational Research. 163, 403-417 (2005)

4. Beynon, M.J.: A Method of Aggregation in DS/AHP for Group Decision-Making with the Non-Equivalent Importance of Individuals in the Group. Computers \& Operations Research. 32(7), 1881-1896 (2005)

5. Beynon, M.J.: The Role of the DS/AHP in Identifying Inter-Group Alliances and Majority Rule within Group Decision Making. Group Decision and Negotiation. 15(1), 21-42 (2006)

6. Beynon, M., Curry, B., Morgan, P.: The Dempster-Shafer Theory of Evidence: An Alternative Approach to Multicriteria Decision Modelling. OMEGA - International Journal of Management Science. 28(1), 37-50 (2000)

7. Beynon, M., Cosker, D., Marshall, D.: An Expert System for Multi-Criteria Decision Making using Dempster Shafer Theory. Expert Systems with Applications. 20(4), 357-367 (2001)

8. Boujelben, M.A., De Smet, Y.: About the application of evidence theory in multicriteria decision aid. In Doumpos, M. and Grigoroudis, E. (Eds) Multicriteria Decision Aid and Artificial Intelligence: Links, Theory and Applications (Google eBook). 161-183 (2013)

9. Dempster A.P.: Upper and lower probabilities induced by a multiple valued mapping. Ann. Math. Statistics. 38, 325-339 (1967)

10. Dezert, J., Tacnet, J.-M., Batton-Hubert, M., Smarandache, F.: Multi-criteria decision making based on DSmT-AHP, BELIEF 2010: Workshop on the Theory of Belief Functions, Brest, France (2010)

11. Du, Y., Han, C., Jing, Y., Liu, J.: TIN-DS/AHP: An Intelligent Method for Group Multiple Attribute Decision Making. Applied Mathematics \& Information Sciences. 8(2), 857-868 (2014)

12. Elgazzar, S.: Enhancing the Company's Financial Performance through Managing the Performance of Supply Chain Operations: A Case Study of an Egyptian Manufacturing Company, Doctoral Thesis, University of Huddersfield (2013)

13. Ennaceur, A., Elouedi, Z., Lefevre, E.: Handling Partial Preferences in the Belief AHP Method: Application to Life Cycle Assessment. In: Pirrone R., Sorbello, F. (Eds.): Ai*IA, LNAI 6934, 395-400 (2011)

14. Ennaceur, A., Elouedi, Z., Lefevre, E.: Reasoning under uncertainty in the AHP Method Using the Belief Function Theory, in S Greco et al (Eds) IPMU, 373-382 (2012)

15. Jiao, L., Liang, Y., Feng, X., Yang, F.: Combining sources of evidence with reliability and importance for decision making. Central European Journal of Operational Research. (2013)

16. Ju, Y., Wang, A.: Emergency alternative evaluation under group decision makers: A method of incorporating DS/AHP with extended TOPSIS. Expert Systems with Applications. 39(1), 1315-1323 (2012) 
17. Liu, B., Tian, Y., Qiu, J. Fire Extinguishing Systems' State Evaluation Basing on Analytic Hierarchy Process and Dempster-Shafer. The 2nd International Conference on Computer Application and System Modeling (2012)

18. Ma, W., Liu, W., Miller, P., Luo, X.: A Game-Theoretic Approach for Preventing Threats in Intelligent Surveillance Systems, in Lomuscio A. et al. (Eds.) Proceedings of the $13^{\text {th }} \mathrm{In}$ ternational Conference on Autonomous Agents and Multiagent Systems, Paris, France (2014)

19. Ma, W., Xiong, W., Luo, X.: A Model for Decision Making with Missing, Imprecise, and Uncertain Evaluations of Multiple Criteria. International Journal of Intelligent Systems. 28, 152-184 (2013)

20. Nedashkovskaya, N.I.: Multi-Criteria Decision Making with Incomplete Expert Information when the AHP is Used, $21^{\text {st }}$ International CODATA Conference (2008)

21. Pankratova, N., Nedashkovskaya, N.: Estimation of Sensitivity of the DS/AHP Method While Solving Foresight Problems with Incomplete Data. Intelligent Control and Automation. 4, 80-86 (2013)

22. Saaty, T.L.: The Analytic Hierarchy Process. McGraw-Hill, New York (1980)

23. Shafer, G.: A Mathematical Theory of Evidence. Princeton University Press, Princeton (1976)

24. Tervonen, T., Lahdelma, R., Salminen, P.: A Method for Elicitating and Combining Group Preferences for Stochastic Multicriteria Acceptability Analysis, TUCS Technical Report (No 638), November (2004)

25. Utkin, L.V., Simanova, N.V.: Multi-criteria Decision Making by Incomplete Preferences. Journal of Uncertain Systems. 2(4), 255-266 (2008)

26. Utkin, L.V., Simanova, N.V.: The DS/AHP Method under Partial Information about Criteria and Alternatives by Several levels of Criteria. International Journal of Information Technology \& Decision Making. 11(2), 307-326 (2012)

27. Wei, G., Liu, J.: A DS/AHP Method for Comprehensive Decision-making in Urban Power System Planning, CICED2008 (2008)

28. Xie, Y., Yi, S., Cao, Y., Lu, Y.: Uncertainty Information Fusion for Flood Risk Assessment Based on DS-AHP Method, IEEE (2011)

29. Yao, S., Guo, Y.-J., Huang, W.-Q.: An improved method of aggregation in DSAHP for multi-criteria group decision-making based on distance measure. Control and Decision. 25(6), 894-898 (2010) 